\title{
El surgimiento, declive y resurgimiento de la evaluación de políticas públicas en el Gobierno americano
}

\section{Guy B. Peters *}

La evaluación de políticas públicas es un aspecto central del proceso de la política. Es crucial por razones utilitarias, proporcionando a los que toman las decisiones, algún tipo de valoración acerca de hasta qué punto los programas que han diseñado y llevado a cabo han sido capaces de alcanzar las metas fijadas para ellos. Pese a que esto no es una tarea fácil, dada la multiplicidad de fines que caracterizan a cualquier programa y dadas las dificultades a la hora de calcular la consecución de esas metas, una investigación de evaluación sistemática proporciona un camino, quizás mejor que cualquier otra vía, para que los encargados de tomar las decisiones sepan lo que han hecho y lo que no han hecho.

La programación y evaluación de políticas públicas también son decisivas para los propósitos de la responsabilidad democrática. Estas actividades proporcionan un medio para determinar qué está pasando con la política y, quizás, una forma de atribuir responsabilidades por sus éxitos o fracasos. La evaluación debería permitir al gobierno ir más allá de los simples conceptos de responsabilidad parlamentaria para precisar los nexos entre las actuaciones y los efectos de éstas. Quizá sea menos importante a la larga hacer responder a un ministro concreto de las actuaciones dentro de su ministerio que comprender lo qué ha ocurrido y por qué.

El gobierno americano (a todos los niveles) ha tendido a invertir cantidades importantes de dinero y tiempo en la evaluación de programas.

De hecho, el momento en que los Estados Unidos desarrollaban su versión del Estado del bienestar en los años sesenta y a principios de los setenta fue también el momento reciente de mayor entusiasmo por la utilidad práctica de las ciencias socia- les. Fue entonces cuando se asumió que las ciencias sociales eran capaces de proporcionar respuestas bastante exactas acerca de lo que hacían los programas y de cuál era la contribución de los programas individuales al éxito general de las intervenciones de la política.

Hubo algunos estudiosos que destacaron las grandes dificultades derivadas de producir ese tipo de información a partir de datos no experimentales (CAMPBELL y STANLEY, 1969); veáse también ALONZO y STARR, 1989), pero fueron ignorados dentro del entusiasmo generalizado que había por las ciencias sociales y la investigación de la evaluación.

Los gobiernos en este momento otorgaron un gran apoyo a la evaluación y, hasta cierto punto, fundaron el desarrollo de todo la industria de "evaluación. Por ejemplo, gran parte de la legislación social aprobada durante ese período requirió extensa evaluación, destinándose por ley hasta un diez por ciento de los fondos de todo el programa a la evaluación. La disponibilidad de ese nivel de fondos indujo a muchas personas a recordar lo mucho que les interesaba la evaluación y lo mucho que sabían a la hora de valorar determinadas áreas de la política. Ese interés puede haber decaído cuando la cantidad de fondos destinada se redujo posteriormente, pero un gran número de estudiantes y profesionales fueron entrenados en ese campo durante ese período y ese campo quedó establecido como una especialidad académica.

Los años sesenta también fueron testigos de la transformación de la Oficina General de Contabilidad de EE.UU. (GAO), que pasó, de ser una organización estrictamente de contabilidad financiera a una institución de evaluación (MOSHER, 1979). Tal y como fue concebida originariamente durante los años veinte, la 
GAO era en sentido estricto una organización postinterventora, es decir, una organización financiera encargada de velar por la legalidad de los gastos federales. El cambio hacia una posición más analítica de la política representó uno de los desarrollos más importantes de la evaluación en los EE.UU. La GAO fue importante, en parte porque ayudó a institucionalizar la idea de que la evaluación era tan importante para la responsabilidad gubernamental como lo era la probidad financiera. La GAO también fue importante porque llevó al desarrollo de varios aspectos de la metodología de evaluación. En esta organización pública, individuos tales como Ray RIST y Elinor CHELIMSKY fueron figuras centrales para el desarrollo de la ciencia de la evaluación, sin duda en EE.UU. y, hasta cierto punto, también alrededor del mundo.

Pese a sus importantes desarrollos en su capacidad analítica, la GAO todavía tiene algunos problemas como institución. Uno de ellos era su confianza en el Congreso para que le guiara sobre qué programas evaluar y qué preguntas hacer. Casi todos los cientos de informes que la GAO emite cada año son elaborados como respuesta a iniciativas del Congreso (normalmente provenientes de un comité) y han limitado la capacidad de la GAO para constituir su propia agenda para revisar. Así, toda la calidad del esfuerzo de la evaluación, aunque no la de los informes individuales, está a merced del abanico de asuntos que conciernen al Congreso y su disposición para hacer frente a dificiles preguntas de políica. Más aún, las peticiones del Congreso tienden a formularse en forma de preguntas bastante modestas, más que en programas enteros, de tal forma que no hay apenas oportunidad de comentar todas las operaciones de todos los programas, ni tampoco con toda seguridad de políticas.

Al igual que por desarrollar una capacidad de evaluación centralizada de la rama legislativa del gobierno, este mismo período se caracterizó por la creación de "tiendas" de valoración independiente en cada organización. Esto fue especialmente cierto respecto de los nuevos planes sociales, económicos y de salud, relacionados con la Great Society. También lo fue hasta cierto punto respecto de ciertos programas que ya habían existido durante algunos años, dada la necesidad de que los programas y las organizaciones parecieran "modernos". También, a finales de los sesenta, fue el período en que los presupuestos para programas estuvieron en boga en el gobierno federal y esta forma de presupuestar tenía requisitos esenciales para la evaluación y comparación de los programas bang for the buck.

Si las agencias querían tener éxito en el proceso presupuestario tenían que ser capaces de justificar que sus programas eran efectivos en el coste e incluso eficientes.

En resumen, aunque los gobiernos siempre habían tratado de encontrar formas de saber si lo que hacían tenía algún impacto demostrable en la sociedad, los años sesenta fueron un período en el que hubo desarrollos académicos e institucionales que comenzaron a hacer esa meta realizable. Tal y como señalaremos más adelante, estos desarrollos no eran una prueba contra las presiones políticas que ponían en duda la utilidad de los programas de gobierno, basados en aspectos ideológicos más que pragmáticos. El siguiente período en nuestra "historia" es un período en el que la capacidad del gobierno para hacer valoraciones razonables de sus actividades está seriamente debilitado.

\section{El declive de la evaluación}

El período que abarca desde la mitad de los años setenta en adelante estuvo caracterizado por un declive en el interés por la evaluación. Este declive en el interés por la evaluación de las políticas públicas pareció estar en función de varios factores. Uno de éstos fue el declive de los recursos disponibles, de tal forma que los gobiernos empezaron a creer que ya no podían permitirse hacer todo aquello que habían hecho en el pasado. Para la organización individual que se encuentra ante el declive - de los recursos, las operaciones de evaluación realizadas eran fáciles candidatos para efectuar recortes. Las "tiendas" de evaluación no proporcionaban servicios directos a los ciudadanos y a menudo daban resultados negativos, así que la organización estaba igual de bien sin ellas.

Una segunda razón que justifica el declive del interés en la política de evaluación es la pérdida de parte del interés académico en esta materia. Tanto si esto se debió a la pérdida del dinero que servía de base a la evaluación, o a la pérdida de confianza en el gobierno ${ }^{1}$, o al cambio de los intereses intelectuales, las universidades y asociaciones profesionales parecían mucho menos interesadas en sus relaciones con las agencias gubernamentales. Un menor número de académicos parecía preocupado por la política e invertía menos de su tiempo académico en estas actividades, tendiendo así la teoría a desarrollarse en direcciones más abstractas (en contraposición a las direcciones más orientadas a la práctica de alguno de los desarrollos anteriores).

Aunque las razones arriba mencionadas eran importantes, la razón más importante que explica el declive de la industria de la evaluación es que el propio gobierno pasó a ser mucho menos que un cliente para la evaluación. Con el advenimiento de la administración Reagan en Washington y de un númiero mayor de gobernadores más conservadores, a nivel estatal, daba la impresión de que los encargados de tomar las decisiones ya sabían las respuestas para su propia satisfacción, y éstas eran que la mayoría de los programas públicos, en realidad, no funcionaban. Si los encargados públicos de la toma de decisiones tienen un fuerte compromiso ideológico de reducir el tamaño y la in- 
fluencia del sector público, apenas tenía sentido invertir recursos adicionales en evaluación.

El periodo de finales de los setenta y ochenta fue testigo del empequenecimiento o eliminación de muchas de las atiendas de evaluación. en agencias federales individuales. Dado que en ese momento había habido muy poca actividad en la elaboración de la política doméstica, esto podría no haber tenido un efecto tan inmediato en la política, pero sí que eliminó de forma efectiva la capacidad para cualquier subsiguiente administración que hubiera querido llevar a cabo un acercamiento más práctico a la política. Curiosamente, aunque ha habido un sustancial renacimiento del interés en la evaluación durante los años noventa, la mayoría republicana en el Congreso continuaba reduciendo las instituciones de evaluación. Redujeron de forma sustancial al personal de la Oficina de Contabilidad General y luego eliminaron la Oficina de Evaluación Tecnológica?

\section{El resurgimiento de la evaluación}

A mediados de los noventa se ha podido observar algún tipo de resurgimiento de la evaluación de políticas públicas en el gobierno americano, especialmente, al nivel federal. Este resurgimiento se ha manifestado de diversas formas y es el resultado de varias medidas legislativas fundamentales, al igual que de algunos cambios en la naturaleza de la administración pública considerada de un modo más genérico. También ha habido un resurgimiento del interés en la evaluación de políticas públicas tanto al nivel estatal como local, pero en esta área la acción principal permanece en el nivel federal.

También ha habido un pequeño pero apreciable aumento en el optimismo elitista (si no público) acerca de la capacidad de los gobiernos en la actualidad para actuar de forma adecuada si están adecuadamente organizados. Aunque Clinton se define a sí mismo como un "nuevo demócrata" $y$, sin duda, no ha sido el prototipo de presidente democrático de «aumentar los impuestos y gastar", aun así, hay algo más de fe en la capacidad del gobierno para solventar, si no todos, al menos algunos de los problemas colectivos. La nueva "fe" en el gobierno depende, en gran medida, del uso de métodos de gestión y evaluación para comprobar la verdadera actuación de cualquiera de los programas puestos en práctica.

Parte del cambio se da, pasando de una nueva pública Gestión a una nueva Pública Gestión. Es decir, la primera ronda de Nueva Gestión Pública ponía el acento en la eficiencia y especialmente resaltaba el minimizar el sector público. Si se gasta menos dinero y hay menos gente encargada de llevar a cabo los servicios, entonces las medidas habituales de eficiencia mejorarán. Sin embargo, de hecho, gran parte de la anterior versión del cambio administrativista no daba especial importancia al cálculo y a la evaluación. Como ataque generalizado a la eficiencia del sector público, se decía que parecía haber una mayor fe en una dirección ideológica, en oposición a una estimación más objetiva de la actuación de los programas del gobierno.

\section{Legislación}

Como ya se ha mencionado anteriormente, gran parte del continuado resurgimiento de la industria de evaluación en el gobierno estadounidenise se puede atribuir a la aprobación de varias medidas legislativas importantes y a la introducción de varias medidas legislativas adicionales por el presidente Clinton. Toda esta reciente legislación propuesta requiere que las agencias gubernamentales mediten acerca de la evaluación y sometan los resultados de sus programas a escrutinio. El grado y tipo de escrutinio depende de las distintas clases de leyes, pero todas ellas requieren un escrutinio sistemático.

\section{La Government Performance and Results Act}

La medida legislativa más significativa que requiere el desarrollo de una mayor capacidad de evaluación es la Government Performance and Results Act de 1993 (GPRA o "Ley de Gestión de Resultados del Gobierno"). Esta ley precedió la elección de la nueva mayoría republicana en 1994 , pero les ayuda de muchas maneras en su agenda ideológica que cuestionaba la utilidad de lọs programas gubernamentales. La GPRA exige que cada organización del gobierno federal desarrolle primero un plan estratégico para su actuación, al igual que un conjunto de medidas asociadas juzgando sus resultados. Se requirió entonces a la Oficina General de Contabilidad para que evaluara esos planes y diera cuenta de ello al Congreso para finales de $1998^{3}$. La mayoría de las organizaciones han llegado ya al momento de exponer planes y la Oficina General de Contabilidad ha empezado a emitir informes con sus opiniones acerca de las propuestas (ver la bome page de la GAO para ver una lista de las actividades concordantes de la GPRA).

El principal propósito de la GPRA era proporcionar a cada una de las organizaciones, al igual que al Congreso, información acerca de los fines establecidos y de la verdadera actua- 
ción de los programas públicos. A diferencia de los amplios acercamientos a la evaluación, propios de los años sesenta, el modelo de evaluación contenido en la puesta en práctica de la GPRA tiende a concentrarse más en desarrollarse a partir de un riúmero bastante limitado de indicadores cuantitativos. En muchos sentidos, esta legislación se identifica más con ideas acerca de la planificación estratégica en los negocios, que con la literatura de evaluación en las ciencias sociales, pero sí que sigue requiriendo organizaciones que piensen con antelación cómo quieren que se evalúen sus resultados.

Se están llevando a cabo intentos por parte de las organizaciones individuales y por parte de la GAO para asegurar que los indicadores seleccionados son los más fuertes y los de más valor, dada su simplicidad, pero tienden a excluirse cuestiones tales como las consecuencias secundarias de los programas y los impactos a largo plazo del análisis ${ }^{4}$. La GAO ha sido en general más bien crítica con los planes y los indicadores que se le han presentado, argumentando que muchos de ellos no se han detallado suficientemente para permitir una verdadera evaluación de los programas (ver, por ejemplo, USGAO, 1997). Por ejemplo, argumentaban que el plan preparado por el Ministerio de Agricultura no:

...contenía los seis elementos requeridos por la "Results Act... La información puesta a disposición es incompleta y a menudo irrelevante, o no estaba directamente relacionada con las metas y los objetivos contenidos en los planes.

Todos los actores en Washington, incluso aquellos miembros del Congreso que motivaron la puesta en práctica de la ley, parecen saber que existe la necesidad de una forma de evaluación más amplia que la que se deduce del GPRA, pero, sin embargo, todavía no han sido capaces de apartarse de las fórmulas más simplistas de evaluación. Intelectualmente hablando, esto no es lo que la mayoría de los profesionales querría encontrar en el ejercicio de una evaluación, pero parece ser lo más posible políticamente dentro del clima actual en Washington. Esta confianza en indicadores simples es también un estilo de evaluación que puede conducir a la toma de decisiones, especialmente presupuestarias, en el Congreso. Le proporciona al Congreso una medida de actuación bastante sencilla, y una medida que puede ser utilizada más adelante en el tiempo para asegurar el progreso.

\section{El Regulatory Review}

Una segunda medida legislativa clave que ha desencadenado algún desarrollo en los mecanismos de evaluación ha sido la
Regulatory Review Act de 1996 (PL-104-208). Esta legislación es otra parte de la agenda republicana para controlar al gobierno y su impacto en la sociedad. La lógica de esta legislación es que la regulación económica y social impone un número de costes a la sociedad, que, normalmente, no se calculan ni se incluyen en la estimación inicial de esas regulaciones. Esta legislación exige, al menos, la identificación de los costes impuestos al sector privado, costes que generalmente exceden con mucho los costes actuales de aplicar las regulaciones'.

La legislación del Regulatory Review requiere que cada nueva regulación sea revisada por la Oficina de Gestión y del Presupuesto y que ésta fije también los costes. Ésta es una forma totalmente económica de evaluación y es más en perspectiva que retrospectivamente, en contraste con la mayoría de los ejercicios de evaluación. Pese a esas diferencias cruciales, esta legislación debería verse como una forma de aumentar la centralidad de la evaluación en el gobierno americano. Se pretende hacer al gobierno considerar las consecuencias de sus acciones y, también, hacerle tomar decisiones basadas en criterios económicos objetivos, al igual que en la idoneidad de un programa u otro, es decir, criterios programáticos. Los criterios económicos son especialmente importantes para los miembros republicanos del Congreso responsables de esta legislación, pero son, sin duda, un elemento importante de cualquier esfuerzo de evaluación.

Asimismo, existe la posibilidad de utilizar esta legislación como base para una evaluación continuada, más que como base para la determinación, de una vez por todas, de los costes. De este modo, se da la posibilidad de que el gobierno continúe determinando los costes de las regulaciones y dé cuenta al Congreso sobre el impacto económico actual de la regulación después de su aplicación. Ésta quedaría como una forma muy limitada de evaluación, pero al menos representaría un nuevo tipo de interés en la evaluación dentro del gobierno federal. Desde Gerald Ford, los presidentes se han preocupado de los costes de las regulaciones y el gobierno y algunas organizaciones de investigación privadas han facilitado estimaciones globales de los costes ${ }^{6}$.

El programa del Regulatory Review está complementado por otra medida legislativa que exige la notificación y la evaluación de mandatos infundados que se imponen al Estado y a los gobiernos locales por el gobierno federal. Otro de los objetivos del programa electoral de los republicanos para EE.UU. consistía en eliminar estos mandatos. En la práctica, no pudieron conseguir la eliminación de los mandatos, pero desde 1995 se exigen los requisitos de la notificación y algún tipo de estimación. La estimación es, en gran parte, un coste financiero del impacto, pero puede aún llegar a convertirse en otra de las formas mediante las que se revitalice la investigación de la evaluación en el gobierno norteamericano. 


\section{La reforma educativa}

Aunque el interés de la Gestión Publica de Clinton en la reforma educativa no está provocando incrementos a gran escala en los esfuerzos de evaluación del gobierno norteamericano, ese interés en la reforma educativa sí que está aumentando de algún modo el interés en esta importante área de políicas públicas. El interés de la Gestión Pública en la reforma educacional es parte de toda una serie continuada de esfuerzos para hacer frente a los fallos de la educación americana ${ }^{7}$. Una diferencia clave de este esfuerzo (y de los que le precedieron) respecto del patrón habitual de la política pública de la educación es que el gobierno federal está asumiendo la responsabilidad principal respecto de un área que históricamente se había reservado a los Estados y a los gobiernos locales.

El programa de Clinton exige el desarrollo de tests a nivel nacional que evalúen la actuación de los estudiantes en diversos niveles de su educación. Al igual que ocurre con muchos programas recientes de tests llevados a cabo por los Estados y por los consejos escolares de cada colegio, se resaltaría el uso de tests estándares, más que el uso de otro tipo de medidas más subjetivas de desarrollo intelectual ${ }^{8}$. Después del resultado de estos tests, se informaría a los colegios individuales. Los republicanos se han opuesto en el Congreso al programa de Clinton, argumentado que la educación es responsabilidad de los Estados y de los gobiernos locales, y no del gobierno federal y que se debería dar la opción a muy distintos tipos de propuestas respecto de la educación.

La idea de este programa de evaluación no es tanto utilizar de forma directa el poder de las organizaciones gubernamentales para corregir las deficiencias observadas, sino más bien movilizar a los padres. Así, si los padres descubren que los colegios de sus hijos no funcionan adecuadamente, pueden hacer uso del poder político para provocar las mejoras ${ }^{9}$. Al utilizar este tipo de propuesta de evaluación y al tratar de remediar las cuestiones, nos encontramos ante problemas clásicos de la evaluación. Por ejemplo, se plantea el riesgo de que los profesores dediquen su tiempo a preparar a los estudiantes para los tests estándar más que para el contenido más genérico del currículum; esto impediría que fueran criticados, ya que dedicarse a hacer realmente su trabajo sería más arriesgado.

\section{La reforma del bienestar social}

Finalmente, la aprobación de la reforma del bienestar social en 1996 impone la necesidad de que haya mayores esfuerzos en materia de evaluación, algunos de los cuales ya están en camino. Esta legislación transfirió la principal responsabilidad en materia de asistencia social al nivel estatal, pero también estableciố una directrices generales al nivel federal, que lievaban a eliminar la asistencia social después de dos años de beneficios continuados, y también otorgó un beneficio máximo de cinco años durante la vida del individuo. Del mismo modo, suprimía la asistencia social a quien no quisiera trabajar ni meterse en un programa de formación para trabajadores.

La reforma del bienestar social ha supuesto uno de los cambios más importantes en el programa social durante años $y$, como consecuencia de ello, es lógico que haya un especial interés en estimar qué pasa realmente una vez que el programa se ha puesto en práctica. Asimismo, a diferencia de lo que ocurría en muchos programas federales anteriores, a los gobiernos de los Estados se les está dando una competencia sustancial para desarrollar los detalles del programa de cada Estado local, con la consecuencia de que se está desarrollando un experimento genuino en la política social por medio este programa. Se da, por tanto, la oportunidad de estimar qué efecto tienen las diferencias del programa en los resultados con (más o menos) similares circunstancias en el ambiente objeto del experimento. Esto también implica que la investigación de la evaluación vuelve a ser el centro del escenario en los Estados y gobiernos locales, tal y como ocurre hasta cierto punto en el gobierno federal.

\section{Conclusión: cambios en la evaluación}

Uno de los cambios más importantes que se dan en la forma en que se dirige la evaluación a todos los niveles de gobierno es la importancia que se da a una evaluación más orientada hacia el consumidor ${ }^{10}$. Es decir, la mayor parte de las propuestas tradicionales de evaluación tenían una orientación de arriba abajo, siendo, bien los profesionales de la evaluación o bien sus clientes en las agencias gubernamentales, quienes tendían a establecer los criterios empleados en la evaluación y que dominaban la evaluación presente. Más aún, había una mayor tendencia a seleccionar un mayor número de indicadores objetivos para las aplicaciones prácticas de la evaluación, en vez de confiar en lo que los consumidores de los servicios gubernamentales opinaban acerca de esos servicios. Muchos profesionales desechaban las opiniones de los destinatarios de los programas como prueba de la calidad de los servicios, por considerarlos poco fiables.

Al menos en Norteamérica, el cambio hacia una mayor adquisición de poder (empowerment) y hacia una mayor participación de los ciudadanos en el gobierno supone que los consumidores ten- 
drán una mayor influencia sobre la evaluación. Parte de esta influencia de los ciudadanos está siendo formalizada a través de informes de los clientes y otros medios similares. A otros aspectos de la óptica de los consumidores se les está haciendo frente, tratando de fortalecer los mecanismos de retroalimentación (ej. bot lines) de tọdo tipo, para que hagan frente a problemas subjetivos u objetivos con los programas y los servicios de puesta a disposición. En otros casos, como se ha señalado anteriormente con respecto a la política de educación, la influencia de los consumidores es algo menos directa, pero aun así importante (ver HOOd, Peters y Wollmann, 1996).
También se da la tendencia casi contraria de la evaluación, de concentrarse más que nunca en los aspectos económicos de los programas y de ignorar los temas del "éxito" o del "fracaso" en el abastecimiento de servicios. Los presidentes republicanos y los legisladores republicanos han tendido a depender del mercado y de los criterios económicos, pero los valores morales del mercado se han extendido. Queda, sin embargo, todavía por ver si la tendencia a dar mayor importancia al público y a los servicios, arriba mencionada, afectará a la forma en que las evaluaciones son dirigidas y al tipo de criterios que dominarán su estimación.
Traducción de Cristina AlBa Mữoz. Revisión de Gemma Sánchez Rontomé.

Universidad de Pittsburgh, EE.UU.

1 Esta pérdida de confianza parece haber precedido los años de la presidencia de Reagan, especialmente con la guerra de Vietnam, que hizo que la incursión académica en el gobieno fuera menos aceptable de lo que lo había sido anteriormente.

2 Esta Oficina también estaba en la rama legislativa y era la que se encargaba de estimar las tecnologías y de aconsejar al Congreso acerca de la innovación en política pública, en política comercial y en otra variedad de asuntos.

"Es algo paradójico que el Congreso haya reducido el tamaño y la capacidad de la Oficina General de Contabilidad pero, a la vez, haya impuesto cargas adicionales a la organización.

" Las evaluaciones de la Oficina General de Contabilidad respecto de las propuestas de la agencia sí que han hecho surgir muchas de estas cuestiones, pero operativamente, tienen que someterse necesariamente a la voluntad del Congreso y tienen que centrarse en indicadores más simplistas.

"En el lenguaje utilizado en EE.UU., el término ‘regulación, hace referencia a todo tipo de legislación secundaria o de desarrollo, pese a ser dictadas y aplicadas por una agencia reguladora.
- El más famoso de éstos es el Centro para el Estudio del Negocio Americano en la universidad de Washington. Durante varios años ha estado liderado por Murray Wiedenham, que fue nombrado por la Administración de Reagan.

- El programa de Clinton es muy similar al impulsado por su predecesor, George Bush. Sin embargo, de forma predecible, ha habido un escaso reconocimiento de esta similitud, por parte del presidente y por parte de los muchos republicanos que ahora se oponen a este plan en el Congreso.

${ }^{s}$ Parte del argumento utilizado en el sistema educacional en los EE.UU. ha sido que los tests estándar no miden aspectos como la creatividad. Así, entienden que los estudiantes de países con puntuaciones altas por encima de la media no serán tan creativos como sus propios estudiantes, algo respecto de lo que parece haber poca o ninguna prueba.

'En los EE.UU., la mayoría de los 14.000 consejos escolares son elegidos por votación, así que al menos queda esta arma para ejercer el control.

${ }^{10}$ Personalmente, detesto el uso del término consumidor, para describir a los ciudadanos que resultan ser los receptores de programas públicos. Aunque sí que reciben beneficios, debería pensarse en ellos en primer término como ciudadanos con derechos políticos.

\section{Bibliografia}

Alonzo, W. y StarR, P. (1989), The Politics of Numbers (New York: Russell Sage).

BerKowTT, D.R. (1991), America's Welfare State: From Roosevelt to Reagan (Baltimore: Johns Hopkins University Press).

CAMPBEL, D.T. y STANLEY, J.C. (1969), Experimental and Quasiexperimental Design for Research (Chicago: Rand-McNally).
Hood, C.; Peters, B.G. y Wollmann, H. (1996), sixteen Ways to Consumerize the Public Sector, Public Money and Management.

MOSHER, F.D. (1979), The GAO: The Quest for Accountability in American Government (Boulder, CO: Westview Press).

USGAO (1997), USDA's Draft Strategic Plan (Washington, DC: US General Accounting Office, GAO/RCED-97-196R). 